\title{
Re-significaciones de los recursos institucionales de gobernanza de la «tercera misión» de las universidades: el caso de los Proyectos de Desarrollo Tecnológico y Social (PDTS) de Argentina
}

\author{
Re-Significations of the Institutional Governance Resources of the «Third \\ Mission» of the Universities: The Case of the Technological and Social \\ Development Projects (PDTS) of Argentina
}

Mauro Alonso'

\section{Resumen}

El presente trabajo se propone analizar un instrumento de gobernanza de la «tercera misión» de la universidad, los Proyectos de Desarrollo Tecnológico y Social (PDTS) en Argentina haciendo foco en los recursos institucionales que pone a disposición de los académicos para promover un mayor uso del conocimiento. Para tal fin, el trabajo se organiza en seis secciones. En primer lugar, se discute la cuestión de la tercera misión de la educación superior en relación a sus posibles definiciones y, en segundo lugar, se historiza la noción de gobernanza de la tercera misión, destacando aquellos conceptos clave que caracterizan la gobernanza de estas actividades. En tercer y cuarto lugar se presentan los elementos contextuales que dan origen al surgimiento del instrumento PDTS. En la quinta sección se presentan aquellos criterios institucionales sobre los que los PDTS introducen resignificaciones, resultando en una gobernanza diferenciada de estas actividades y finalmente, en la última sección se revisitan algunas de las discusiones. El principal emergente del análisis realizado, muestra, para el caso argentino, cómo los PDTS - como instrumento — incorpora algunas nuevas consideraciones en materia de gobernanza de actividades de tercera misión que, o reconocen matices novedosos respecto de las definiciones de estas actividades o resignifican algunos de los elementos tradicionales de la práctica académica.

\section{Palabras clave}

Tercera misión de la universidad, gobernanza, uso del conocimiento, PDTS.

\section{Abstract}

This paper aims to analyze a governance instrument for the university's «third mission», the Technological and Social Development Projects (PDTS) in Argentina, focusing on the institutional resources that it makes available to academics to promote greater use of knowledge.

The focus of the work involves a literature review regarding the concepts presented together with an empirical analysis nourished by documentary sources and interviews with actors involved in the management of the instrument. For this purpose, the work is organized into six sections. In the first place, the question of the third mission of higher education is discussed in relation to its possible definitions and, secondly, the notion of governance of the third mission is historicized, highlighting those key concepts that characterize the governance of these activities. In third and fourth place, the contextual elements that give rise to the emergence of the PDTS instrument are presented and the origin, definitions and scope of the instrument are characterized. Finally, the fifth section presents those institutional criteria on which the PDTS introduce resignifications, proposing a differentiated governance of these activities. Finally, in the sixth section some of the rehearsed discussions are recovered.

\section{Keywords}

Third mission of the university, governance, use of knowledge, PDTS.

\section{Cómo citar/Citation}

Alonso, Mauro (2021). Re-significaciones de los recursos institucionales de gobernanza de la «tercera misión» de las universidades: el caso de los Proyectos de Desarrollo Tecnológico y Social (PDTS) de Argentina. Revista de Sociología de la Educación-RASE, 14 (2), $205-227$. http://dx.doi.org/10.7203/RASE.14.2.18128. 


\section{Introducción. La(s) misión(es) de la universidad en cuestión}

Desde hace varias décadas, el término «tercera misión» (o tercera función) ha ido adquiriendo cada vez más atención e importancia tanto en la propia práctica de los académicos como en los ámbitos de gestión de la política científica y universitaria y aparece, en tanto declamación normativa, como una función diferenciada de las dos originarias (Trencher et al., 2014; Etzkowitz, 1990). Esta tercera misión —más allá de la ausencia de una definición taxativa- y producto también de los múltiples sentidos construidos respecto de su posible definición, es aquella que de forma más explícita se propone que el conocimiento que se produce en la universidad contribuya, en algún sentido, al contexto social en el que esta se encuentra, de manera que se vuelva observable en algún tipo de vínculo específico con su entorno. A lo largo de la historia de la universidad, esta función ha sido nombrada de múltiples formas y los distintos modelos o visiones de universidades dieron diferentes respuestas y denominaciones al vínculo de las universidades con las sociedades: extensión, transferencia, misión o función, compromiso social, impacto social, vinculación, etc.

Por cierto, estas formulaciones que podríamos llamar polisémicas según el contexto en el cual las encontremos, como también opuestas en sus sentidos o con acentos puestos en aspectos diversos, dan cuenta de una misma función histórica (o primigenia) que pone blanco sobre negro aquello que se espera de la universidad como institución de la vida social.

Veamos esta multiplicidad de definiciones más en detalle. La Unidad de Investigación de Política Científica (SPRU por sus siglas en inglés) de la Universidad de Sussex del Reino Unido define estas actividades o tercera misión como aquellas que «se refieren a la generación, uso, aplicación y explotación de conocimiento y otras capacidades de la universidad fuera del ambiente académico» (Molas-Gallart et.al., 2002: 3). Otras definiciones varían desde descripciones muy acotadas como «vínculos universidad-sociedad» (Sánchez-Barrioluengo 2014: 176) hasta caracterizaciones complejas que incluyen «todas las actividades que incluyan producción, comunicación, difusión o transmisión de conocimiento hacia el entorno» (Molas-Gallart and Castro-Martínez, 2007: 321) o «actividades relacionadas con la investigación (transferencia de tecnología e innovación), educación (formación docente) y vinculación con la sociedad (visitas a museos, conciertos, conferencias, trabajos de voluntariado, consultoría, etc.)» (E3M Project 2012: 6) o «la relación de la universidad con el mundo no-académico: industria, policymakers y sociedad» (Schoen, A. et al., 2006: 127) para mencionar algunas de las nomenclaturas.

Al analizar en detalle los sentidos construidos a los que refieren las diferentes interpretaciones y dominios de la tercera misión, se pueden deducir tres componentes principales de las actividades: comercialización y explotación económica del conocimiento generado académicamente, utilización del conocimiento teórico y transferencia de conocimiento de las universidades a la sociedad y las políticas públicas (Molas-Gallart et al., 2002; Trencher et al., 2014; Laredo, 2007).

Al diseñar los mecanismos para alcanzar objetivos de su tercera misión — dentro de las tres dimensiones referidas-, una universidad puede verse tentada a centrarse en la primera opción. La lucha por el presupuesto producto de la inflación y la reducción del financiamiento público, a menudo deviene en la necesidad de encontrar fuentes alternativas de financiamiento. La explotación económica de los resultados de la investigación producidos dentro de la institución puede ser una forma efectiva de asegurarse aportes económicos. Esta estrategia incluye patentes, copyright y licencias, la creación de spin-offs o empresas mixtas de base tecnológica (Molas-Gallart, 2002). Sin embargo, la cantidad de fondos que se puede recaudar 
generalmente es limitada en casi todas las disciplinas científicas, excepto en biotecnología e ingeniería de software (Laredo 2007) y se vuelve incluso más acotado para el caso del contexto latinoamericano ${ }^{2}$.

La segunda opción describe la búsqueda de (nuevos) campos de aplicación para el conocimiento teórico. Los resultados de la investigación a menudo no están directamente relacionados con problemas específicos de los contextos de aplicación —en especial al referirnos a la investigación básica en particular-. Aquí aparece una consideración de transferencia de conocimiento que supone que los resultados de la investigación pueden resignificarse como aplicables, tanto en campos prácticos como de otras disciplinas. Esta búsqueda puede estar impulsada por objetivos idealistas, en parte como respuesta a un compromiso social de los propios académicos, puede dar un nuevo sentido a los investigadores (Di Bello, 2013; Vaccarezza y Zabala, 2002) resaltando que los investigadores también pueden obtener nuevos conocimientos de los campos prácticos de aplicación consolidando una dinámica recursiva del proceso de investigación (Olmos-Peñuela, 2015). El proceso de transferencia de conocimiento en la búsqueda de campos de aplicación a veces se puede combinar con los beneficios financieros como se describió anteriormente, pero es importante distinguir entre un interés primario en la búsqueda de «usabilidad» (Fischman, 2014) del conocimiento y el interés primario en el rédito económico (Hayden, Weiß, Pechriggl, y Wutti, D., 2018). Discutiremos estas consideraciones, hasta aquí de orden general, en las secciones siguientes.

Finalmente, la última opción tiene una connotación diferente de las dos primeras y puede verse, de algún modo, como una obligación moral o un deber cívico o ético (Hayden, Petrova y Wutti, 2018). Las universidades juegan un papel importante en la comunicación y la sensibilización del público sobre los hallazgos científicos (Hayden, Petrova y Wutti, 2018). Por otro lado, se asume, o se espera que, los tomadores de decisiones en los ámbitos políticos debieran basar sus decisiones en el estado actual del conocimiento científico, de modo que se espera que los investigadores brinden a estos interesados el conocimiento necesario para la toma de decisiones en materia de diseño de políticas públicas (Weiss, 1989; Jasanoff, 2003) y aún más, que traduzcan o resignifiquen esa información para contextos específicos.

En cualquier caso, como se observa, hemos hecho referencia a cada sentido posible de la actividad como una operación de transferencia de conocimiento. Recuperando las conceptualizaciones antes vertidas, reconocemos al conocimiento como el principal producto de la práctica universitaria, intentando nomenclar las múltiples actividades que incluyen el sentido de generación y uso al que nos hemos referido hasta aquí.

Introducimos en la sección siguiente algunas consideraciones respecto de la gobernanza de la tercera misión que nos permiten, en primer lugar, historizar los cambios no solamente en la forma en la que las instituciones de educación superior nombran y jerarquizan sus misiones sino también en que actividades resultan de legítimas o se reconocen legítimas por parte los ámbitos de gestión.

El presente trabajo analiza un instrumento de gobernanza de la «tercera misión» de la universidad, los Proyectos de Desarrollo Tecnológico y Social (PDTS) en Argentina haciendo foco en los recursos institucionales que pone a disposición de los académicos para promover un mayor uso del conocimiento.

El trabajo revisita literatura relevante sobre los conceptos presentados junto con un análisis empírico nutrido de fuentes documentales y entrevistas a actores involucrados en la gestión del instrumento. La re-

2 Sobre las particularidades del contexto latinoamericano, véanse trabajos seminales como Naidorf (2009) y Naidorf y Perez Mora (2012) que analizan las características de la cultura académica y las denominadas actuales condiciones de producción intelectual en diversos países de Latinoamérica. 
visión documental se nutrió de un corpus de documentos oficiales del Ministerio de Ciencia, Tecnología e Innovación productiva de Argentina (12 documentos oficiales del período 2010-2016) y entrevistas a policy-makers vinculados a la implementación del instrumento PDTS analizado ( 9 entrevistas en profundidad realizadas entre 2017 y 2020). El diseño metodológico (eminentemente cualitativo) que orientó la construcción empírica estuvo orientado a indagar en las definiciones sobre transferencia y uso de conocimiento en los lineamientos de política científico-tecnológica tanto en documentos oficiales como en la voz de los funcionarios entrevistados.

En la siguiente sección se discute la cuestión de la tercera misión de la educación superior en relación a sus posibles definiciones y se historiza la noción de gobernanza de la tercera misión, destacando aquellos conceptos clave que caracterizan la gobernanza de estas actividades.

\section{Gobernanza de la tercera misión}

Producto de la profesionalización de las actividades académicas durante el siglo xx, se intensifica, jerarquiza y compartimenta la gestión de las misiones dentro de la gestión de las instituciones de educación superior (Benneworth, 2014). Como vimos, incluso cuando las «tres misiones» están intrínsecamente vinculadas, haremos foco en aquellas que, en sentido amplio, refieren a la movilización del conocimiento académico (Naidorf et al., 2019), producido en la universidad por sus científicos, hacia sus entornos. Dicho de otro modo, los productos del proceso de producción de conocimiento con objetivo de apropiación (resignificación) por parte de agentes no académicos, o extra-académicos.

En un artículo titulado Advancing the Science of Science and Innovation Policy, Daniel Sarewitz (2010), propone una distinción para el diseño de los objetivos de gestión de la investigación académica. El autor distingue entre productos (outputs) y resultados (outcomes), de dicha práctica. Desde la consagración del modelo lineal-ofertista en el informe de Bush, Ciencia: la frontera sin fin de 1945, la distinción entre productos y resultados de la ciencia supuso un solapamiento (Gordon, 2011). A partir de entonces, el modelo hegemónico para el diseño de la política científica en los países centrales y luego en América Latina pasó a basarse en una concepción insumo-producto, donde el Estado debía garantizar los recursos para el funcionamiento de la ciencia (principalmente básica), de la que se derivarían naturalmente las investigaciones aplicadas y los desarrollos tecnológicos que resultarían en beneficios sociales y económicos (Gordon, 2011). El modelo insumo-producto supone que al sistema científico hay que garantizarle ciertos insumos (inputs) (financiamiento, recursos humanos), para derivar resultados (outputs) mensurables (publicaciones, patentes, etc.). Para este esquema no existe una preocupación por los resultados sociales de la investigación porque se supone un fin inexorable, cuasi natural: los beneficios de la ciencia fluyen de manera hacia la sociedad sin la necesidad de intermediaciones o instrumentos para gobernar dicha circulación. De este modo, la gobernanza de estas actividades debiera solamente ceñirse a la provisión de recursos en la espera de productos socialmente valiosos que encontrarán su camino hacia otros ámbitos de la vida social.

Ya pasado el período de institucionalización de la práctica científica, durante la primera mitad del siglo $\mathrm{xx}$, comienza a consolidarse, decíamos, un creciente reclamo por la intervención de la universidad en el mundo social que ponga de manifiesto el cumplimiento de su «función social» (Polanyi, 1968). La masificación de la educación superior, la mayor escala (y alcance) de la investigación universitaria y el papel más importante del conocimiento en el proceso de producción económico han transformado a pequeñas instituciones de élite, gestionadas por pares académicos, en grandes organizaciones con múltiples tareas que 
necesitan de nuevas estructuras de gobernanza para gestionar todas las tareas y roles que existen en las instituciones. Zaharia y Gibert (2005) sugieren que ese reclamo proviene de la necesidad de crecimiento constante en la sociedad basada en el conocimiento, que «depende de la producción de nuevo conocimiento» y es el conocimiento el principal output de las universidades. Por cierto, sostienen, que este «nuevo conocimiento» debería transmitirse a través de la educación y las nuevas tecnologías de comunicación y garantizarse su «utilización en nuevos procesos o servicios industriales» (Zaharia y Gibert, 2005: 31). En este sentido, Robertson (2000) identifica un posible alejamiento de «la producción de conocimiento basado en disciplinas académicas construidas institucionalmente (por los propios académicos) hacia formas de producción basadas en la aplicación del conocimiento a problemas específicos en entornos sociales, económicos y comerciales específicos» (Robertson, 2000: 48). La consolidación de la tercera misión en las universidades está, entonces, indisolublemente unida a la necesidad creciente de que las universidades demuestren su propósito en la sociedad. Las universidades ya no pueden señalar el valor intrínseco de la educación, ahora deben demostrar su utilidad para promover mejoras materiales en la sociedad (Lockett, Wright, y Wild, 2008) y, de este modo, el aumento de las actividades de tercera misión ha presentado a las universidades y al Estado un nuevo medio para hacerlo: un nuevo escenario en el que probar su valor (Lockett, Wright y Wild, 2008).

Un aspecto evidente de esta transformación se observa también en la gobernanza de las actividades de transferencia de conocimiento. Las universidades siempre han estado involucradas en actividades de transferencia de conocimiento; no son algo nuevo, «descubierto» en los últimos años, como se argumenta desde algunas tradiciones cómo Triple Hélice o «Modo 2» (Etzkowitz y Leydesdorff 2000; Gibbons et al., 1994) que proponen la idea de una nueva revolución académica que se caracteriza por nuevas universidades que producto de estos cambios se involucran, ahora, en actividades de transferencia. Podemos rastrear las interacciones entre los profesores universitarios (no necesariamente las universidades mismas) y las empresas, desde el desarrollo de la industria química en el siglo XIx (Meyer-Thurow, 1982; Merton, 1937).

Sin embargo, lo nuevo es la institucionalización de los vínculos entre la universidad y la sociedad a través de la participación directa de la universidad. Sería incorrecto hablar de una revolución académica porque una verdadera «segunda revolución académica» (la primera es la introducción de la investigación junto con la enseñanza a principios del siglo XIX ${ }^{3}$ ) implicaría que todo el personal de una universidad esté involucrado tanto en investigación como en enseñanza, y en actividades de tercera misión, lo que está lejos de la realidad en la mayoría de las universidades de todo el mundo ${ }^{4}$. Si bien parte del personal académico está muy involucrado con usuarios, organizaciones del tercer sector o empresas a través de las oficinas o secretarías universitarias especializadas en transferencia de conocimiento, un número significativo se ocupa principalmente de la enseñanza y la investigación, incluso cuando pueda realizar alguna vinculación ocasional. El cambio sustantivo está en el tipo de actividades realizadas por el personal académico (en respuesta a nuevas demandas) y su importancia o peso relativo respecto en el conjunto de las actividades académicas, poniendo de manifiesto no solo la necesidad de apoyarlas, gestionarlas y organizarlas de una manera eficiente sino de promoverlas.

\footnotetext{
3 Me refiero a la transformación Humboldtiana. Véase (Etzkowitz, 1990).

4 Quizás esto se observe de forma menos evidente para el caso de Universidad Latinoamericana. Al respecto, corresponde mencionar la tradición de extensión universitaria ya presente en la reforma de 1918 (Naidorf, Perrotta y Cuschnir, 2020).
} 
Estos cambios han traído la necesidad de un sistema de gobernanza que debe hacer frente tanto al mayor tamaño y complejidad de la universidad como a su producción grupal altamente específica y diversificada (Geuna and Muscio, 2009). Algunos países están reconsiderando los roles de sus instituciones de investigación (y su financiamiento de investigación) dentro de los sistemas nacionales de innovación, y especialmente con respecto a los institutos de investigación, junto con los cuales hay presiones políticas crecientes para las universidades a recaudar fondos de investigación de la industria y contribuir activamente al desarrollo económico. Esto se debe a que se considera que la investigación universitaria puede promover la difusión de conocimiento situado (Boroschi y Lissoni ,2001; Calderini y Scellato, 2005) y conducir a procesos regionales de innovación (Jaffe 1989). En principio, ayudar a la transferencia y comercialización de resultados es de interés tanto para los investigadores como para la sociedad (Litan et al., 2007) y la transferencia de conocimiento se ha convertido en un problema estratégico: es una fuente potencial (aunque incierta) de financiación para la investigación universitaria y se ha convertido en una herramienta política para el desarrollo económico. Los trabajos que postularon una mayor influencia del contexto de aplicación como orientador de las actividades académicas (Gibbons et al., 1994) y aquellos vinculados al estudio de los sistemas nacionales de innovación (Lundvall, 1992; Nelson \& Rosenberg, 1993) estuvieron en el centro de los debates sobre el rol de la universidad en la capacidad innovadora de las naciones y en su nivel de desarrollo productivo.

Sin embargo, este proceso también fue significado de forma crítica, siguiendo a Naidorf (2009), como mercantilización de la educación superior y por suponer, como vimos, una clara «apertura» de la universidad hacia el mercado, explicitándose en las formas de vinculación universidad-empresa (Naidorf, 2005) donde se vuelve visible una valoración de la práctica académica por su capacidad de ser «usada» por la empresa (Naidorf, 2016). En efecto, esta tensión creciente hacia una noción utilitaria —en términos de rentabilidad económica, estrictamente mercantiles — de la práctica académica derivo en dos fenómenos en distintos niveles que motivan el foco de este trabajo.

En primer lugar, como dijimos, la intensificación, por parte de los ámbitos de gestión de las universidades para con los académicos a involucrarse en actividades de transferencia de conocimiento en ocasiones no reconoce los propios matices de estas dinámicas. Si la tercera misión se consolida, en especial en términos económicos, como beneficiosa para la institución, entonces ésta es ahora reclamada a los académicos y esta función no puede ser reducida solamente a la dimensión de la comercialización económica: existen múltiples actividades vinculación entre academia y sociedad que exceden el binomio universidad-empresa. En segundo lugar, y solo analíticamente escindible de la anterior, producto de que esta nueva demanda no pareciera tener en cuenta esas múltiples dinámicas internas de aquello que conocemos como academia — disciplinas, campos de conocimiento, prestigio, trayectorias, etc. - comenzó a proliferar la producción académica respecto de qué es, cómo se realiza y quiénes intervienen en aquellas actividades de tercera misión, ofreciendo múltiples formas y modelos para su estudio, implementación y evaluación de resultados.

De modo que: si la transferencia y vinculación se vuelve a ahora explícitamente reclamadas a los académicos, debieran entonces también reconocerse modalidades de estas dinámicas por fuera de aquellas que sólo observan la transferencia tecnológica o los vínculos universidad-empresa y ponerse además recursos (simbólicos y materiales, en términos de Bourdieu) desde las instituciones de educación superior a fin no solo, de definir aquellas actividades que reconocen como deseables sino también para promoverlas. 
En efecto, esto aparece como el principal emergente del análisis realizado. En tanto pesa cada vez más en la cultura de investigación en las universidades la necesidad de transferir conocimiento hacia la sociedad, se ponen en agenda nuevos instrumentos de gestión de las actividades académicas que reconocen estrategias de transferencia y vinculación no tradicionales (como patentes, consultorías, creación de start-ups, spin-offs, etc.) que reconocen nuevas dinámicas de vinculación y colaboración con otros agentes sociales por fuera del campo académico.

La sección que sigue, muestra, para el caso argentino, el recorrido realizado por el complejo científico y tecnológico nacional que pone en evidencia este componente coyuntural en el que se reclama de forma más explícita a los académicos la promoción de estas actividades producto de un período previo de fortalecimiento sostenido del propio sistema de ciencia y tecnología. Luego de este período, sostengo, los PDTS aparecen como un instrumento que incorpora algunas nuevas consideraciones en materia de gobernanza de actividades de tercera misión que o reconocen matices novedosos respecto de las definiciones de estas actividades o resignifican algunos de los elementos tradicionales de la práctica académica.

\section{El Caso de los Proyectos de Desarrollo Tecnológico y Social (PDTS) en Argentina. El contexto político que dio origen al instrumento}

Durante el gobierno de Néstor Kirchner (2003-2007) se designó al Ing. Tulio del Bono al frente de la Secretaría de Ciencia y Técnica de la Nación (SECYT), y al bioquímico Lino Barañao a cargo de la Agencia Nacional de Promoción Científica y Tecnológica (ANPCYT). El nuevo secretario creó un Observatorio de Ciencia, Tecnología e Innovación, al que le encargó la elaboración de las Bases para un Plan Estratégico de Mediano Plazo en Ciencia, Tecnología e Innovación, que serían incorporadas en el Plan Estratégico Nacional de Ciencia, Tecnología e Innovación Bicentenario 2006-2010. Las Bases fueron presentadas como un ejercicio de prospectiva, elaborado a partir de un trabajo participativo que involucró a investigadores, empresarios de diversos sectores y funcionarios de distintas áreas de gobierno (Albornoz y Gordon, 2011). El Plan establecía una serie de objetivos estratégicos y metas cuantitativas y cualitativas a ser alcanzadas entre 2010 y 2015. Entre sus objetivos figuraban:

1. Objetivo Estratégico 1: orientación de la I+D hacia un mayor conocimiento de los problemas de la sociedad, la mejora de la calidad de vida y el desarrollo social.

2. Objetivo Estratégico 2: creación y aplicación de conocimiento para la explotación responsable de los recursos naturales, protegiendo el ambiente.

3. Objetivo Estratégico 3: fortalecimiento de la innovación, la modernización tecnológica y la vinculación tecnológica en la producción industrial y agropecuaria.

4. Objetivo Estratégico 4: aumento de la base científica y de la capacidad tecnológica (PENCTI Bicentenario, 2006).

Según Albornoz y Gordon (2011), las metas cuantitativas a alcanzar en el transcurso de los siguientes diez años, como condición necesaria para el logro de las metas cualitativas, habían sido las siguientes: 1) La inversión total del país en I+D debía alcanzar el 1\% del PBI; 2) la inversión privada en I+D debía equiparar a la inversión pública; 3) el número de investigadores y tecnólogos debía equivaler a un 3\% de la PEA, y 4) las diecinueve provincias que concentraban alrededor del $20 \%$ de los recursos de I+D, debían duplicar su participación en el total. La institucionalización de las Bases en el Plan Bicentenario, supuso 
la redefinición de las áreas prioritarias en Áreas-Problemas-Oportunidad —en consideración de problemáticas de desarrollo productivo—, y en Áreas-Temáticas-Prioritarias — según consideraciones disciplinarias y tecnológicas. Estos dos grandes conjuntos de áreas prioritarias fueron, a su vez, desagregados en ochenta y dos líneas prioritarias. Sin embargo, se ha señalado que tal cantidad atentó contra la factibilidad de poder priorizar una determinada línea, diluyendo así su carácter prioritario (Emiliozzi, 2011).

La revisión de criterios de relevancia y pertinencia también habían sido puestos en cuestión y revisados teóricamente en Naidorf et al., (2007) y Naidorf (2011) donde se analizó el plan Bicentenario y el impacto que este tuvo en la definición de agendas de investigación en 6 universidades nacionales.

\section{1. Crecimiento del complejo científico y tecnológico argentino}

La cantidad total de investigadores y becarios del país era de 41741 en el año 2000, considerando a los miembros del CONICET, universidades públicas y privadas, institutos públicos de investigación y empresas (RICyT, 2013). Cuando este valor se corrige según las dedicaciones equivalentes a jornada completa (EJC) —despejando así las dedicaciones parciales_, este número se reduce a 26420 (Gordon, 2015). Entre 2000 y 2001, se redujo en aproximadamente 1300 la cantidad de investigadores, producto de la crisis que forzó a la emigración o la búsqueda de otras inserciones profesionales. A partir de entonces, comenzó un proceso de crecimiento sostenido (Naidorf, Perrotta, 2016). La cantidad total de investigadores y becarios (personas físicas), aumentó a una tasa promedio anual ponderada de 5,90\% entre 2000 y 2010, mientras que su EJC aumentó el 6,06\%. Esto indica que no sólo se incorporaron nuevos investigadores, sino que también se mejoró la dedicación de los mismos. Este aumento sostenido permitió alcanzar, en 2010, los 2,88 investigadores EJC cada mil personas de la población económicamente activa (PEA), acercándose así al objetivo trazado en el Plan Bicentenario (2006-2010), de contar con 3 investigadores EJC cada mil personas de la PEA para 2010.

Con la llegada al gobierno de Cristina Fernández de Kirchner en diciembre de 2007, fue creado el Ministerio de Ciencia, Tecnología e Innovación Productiva (MINCTIP), designándose a Lino Barañao —hasta entonces Presidente de la ANPCYT_ al frente del mismo. La creación, por primera vez, de un Ministerio de Ciencia y Tecnología (e Innovación Productiva), fue celebrada por la propia comunidad científica como un indicador del otorgamiento de mayor prioridad política a la actividad científica, a la vez que supuso la integración al Gabinete Nacional de un Ministro de esta área.

En cuanto a la organización institucional, estas innovaciones supusieron la separación de la política universitaria de la investigación científica, por cuanto la Secretaría de Políticas Universitarias continuó bajo la órbita del Ministerio de Educación de la Nación. El nuevo Ministerio creó dos Secretarías: una de Políticas y Planeamiento y otra de Articulación, destinada, justamente, a coordinar políticas con el conjunto del sistema científico. A su cargo quedó el Consejo Interinstitucional en Ciencia y Tecnología (CICYT), que venía funcionando en la órbita de la SECY'T desde su creación, a través de la Ley N. ${ }^{\circ}$ 25467 de Ciencia, Tecnología e Innovación de 2001 (Gordon, 2015).

Un indicador de la jerarquización de la actividad científica es el aumento de la participación de la función ciencia y técnica en el presupuesto nacional, que pasó de 1,38\% en 2003 a 1,78\% en 2007, hasta llegar a 1,94\% en 2010 (RICYT, 2015) significando un aumento del 40\% entre 2003 y 2010. Más importante aún es que se trata de una mayor participación de la función ciencia y técnica en un presupuesto que aumentó un 412\% entre 2003 y 2010 en términos nominales. Sin embargo, la partici- 
pación del MINCTIP (incluida la ANPCYT, su agencia de financiamiento) sobre el total de la función ciencia y técnica, permaneció en torno al 16\% entre 2005 y 2010. Esta circunstancia indica que la asignación de rango ministerial al organismo encargado de establecer la política de ciencia y tecnología, no fue acompañada de un aumento de su poder sobre la distribución de recursos económicos, ya que su participación relativa dentro del presupuesto para el área permaneció estable (Albornoz y Gordon, 2011). Por lo tanto, y a fin de poder avanzar con políticas implícitas de desarrollo tecnológico sectorial, resulta necesario buscar mecanismos de coordinación junto con los organismos de ciencia y tecnología sectoriales.

En relación a los instrumentos adoptados, en los últimos años el MINCTIP ha intentado avanzar hacia una mayor focalización de las políticas, en cuanto a la identificación de beneficiarios e impactos potenciales. En este sentido, se trata de avanzar más allá de las políticas horizontales implementadas desde las reformas institucionales de los años noventa (Gordon, 2011, 2015), que se caracterizaron por el abandono de las políticas sectoriales de desarrollo tecnológico, en línea con los enfoques a favor del desmantelamiento de las instancias estatales de definición de prioridades.

\subsection{Características distintivas de los Proyectos de Desarrollo Tecnológico y Social (PDTS)}

Desde el año 2011, las autoridades del Ministerio de Ciencia, Tecnología e Innovación Productiva de la Nación (MINTIP) pusieron en un lugar central de la agenda política la revisión de las prácticas de evaluación, como un aspecto más del proceso de transformación del sistema de ciencia y tecnología que buscaba aumentar la cantidad de investigación aplicada y desarrollo tecnológico que se realiza en el país.

Dentro de la órbita del MINCTIP, este desafío estuvo a cargo de la Secretaría de Articulación Científico Tecnológica (SATC), por entonces en manos del Doctor Alejandro Ceccatto, quien a su vez presidía el Consejo Interinstitucional de Ciencia y Tecnología (CICYT). Este último nuclea a los principales organismos públicos que realizan investigación: la Administración Nacional de Laboratorios e Institutos de Salud (la ANLIS), el Consejo Interuniversitario Nacional (el CIN) por las universidades nacionales, la Comisión Nacional de Actividades Espaciales (la CONAE), el Consejo de Investigaciones Científicas y Técnicas (el CONICET), la Comisión de Energía Atómica (la CNEA), el Consejo de Rectores de Universidades Privadas (el CRUP), el Instituto Antártico Argentino, el Instituto Nacional del Agua (el INA), el Instituto Nacional de Desarrollo Pesquero (el INIDEP), el Instituto Nacional de Tecnología Agropecuaria (el INTA), el Instituto Nacional de Tecnología Industrial (el INTI), el Ministerio de Defensa, la Comisión de Investigaciones Científicas (la CIC) del Gobierno de la Provincia de Buenos Aires, la Secretaría de Políticas Universitarias, incluyendo su Programa de Incentivos, y la Comisión Nacional de Evaluación y Acreditación Universitaria (la CONEAU).

La primera acción tomada por las autoridades fue la organización de un taller de discusión entre todas las instituciones científicas nucleadas en el CICYT. El encuentro se tituló «1 ${ }^{\text {er }}$ Taller de evaluación del personal científico y tecnológico para una Argentina Innovadora», tuvo lugar en Buenos Aires en septiembre de 2011 y se centró en la necesidad de revisar mecanismos institucionales que permitan promover investigaciones orientadas y aplicables más cercanas desarrollo de tecnologías y resolución de problemas concretos. 
Como resultado de la labor de la Comisión Asesora, surgieron en 2012: el «Documento I de la Comisión Asesora sobre Evaluación del Personal Científico y Tecnológico del MINCYT: Hacia una redefinición de los criterios de evaluación del personal científico y tecnológico» y el «Documento II de la Comisión Asesora sobre Evaluación del Personal Científico y Tecnológico: Precisiones acerca de la definición y los mecanismos de incorporación de los Proyectos de Desarrollo Tecnológico y Social (PDTS) al Banco Nacional de Proyectos del MINCYT (2012)».

Uno de los ejes centrales de la discusión fue la necesidad de precisar qué se entiende por investigación aplicada u orientada, qué por desarrollo tecnológico y cómo promover investigaciones socialmente útiles. La particularidad de los PDTS ha sido el intento por articular mecanismos de evaluación para proyectos que tengan en cuenta ambos criterios explicitados pero que a la vez incorporen también el uso concreto de los resultados esperados sin dejar de asumir que los mismos son proyectos de investigación y deben propender a la generación de conocimiento original, lo que será objeto específico del análisis en las secciones sucesivas. En este sentido, se diferencian en un primer nivel analítico de proyectos de investigación aplicada puesto que no solamente se busca el desarrollo de mecanismos y capacidades para la aplicación de un conocimiento previamente generado, sino también la generación de un nuevo producto original que ha de ser adoptado mediante el reconocimiento de un contexto de producción de conocimiento específico y delimitado. A su vez, se buscó ofrecer una resolución que incorpore una visión más específica de la noción de originalidad y que retome e incorpore la aplicabilidad del conocimiento como un eje sustantivo.

En palabras del Doctor Hugo Sirkin, ex secretario de Ciencia y Técnica de la Universidad de Buenos Aires, los PDTS: «Son un intento para equilibrar el desarrollo científico que tiene el país, que podríamos decir que es desparejo. La argentina tiene una tradición científica relativamente importante, un desarrollo fuerte en un conjunto grande de especialidades y una base muy sólida para la actividad científico técnica. Pero tiene cierto sesgo que es producto de la historia, por cómo se desarrollaron estas actividades a lo largo de la última parte del siglo pasado» ${ }^{5}$.

Según el Documento II de la Comisión Asesora sobre Evaluación del Personal Científico y Tecnológico: precisiones acerca de la definición y los mecanismos de incorporación de los Proyectos de Desarrollo Tecnológico y Social (PDTS) al Banco Nacional de Proyectos del MCTIP un PDTS:

a. Consiste en un proyecto de actividad que hace uso de conocimientos científicos y tecnológicos pertenecientes a una o más disciplinas;

b. Está compuesto por elementos de distintos tipos (tales como antecedentes teóricos, metodologías y técnicas, información específica, fases, recursos técnicos y financieros, experticias, legitimidad ética y social, criterios evaluativos de la misma actividad) suficientemente explícitos y ordenados de manera que permitan la comprensión de sus fines y objetivos, el alcance del avance cognitivo propuesto, la factibilidad de su realización, la evaluación de su gestión, avance y logros;

c. Tiene por objetivo la resolución de problemas o necesidades de carácter práctico; esto es, problemas y necesidades no justificados en la sola curiosidad científica, el avance del conocimiento

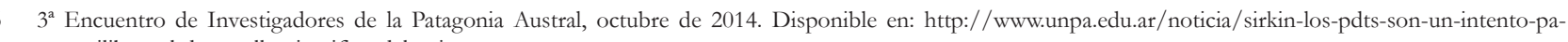
ra-equilibrar-el-desarrollo-cientifico-del-pais. 
disciplinar o la solución de incógnitas teóricas, sino problemas o necesidades enmarcados en la sociedad, la política, la economía o el mercado;

d. Está orientado a la resolución de un problema o al aprovechamiento de una oportunidad — sea ésta una tecnología, un marco normativo, un programa de intervención en la sociedad, una prospectiva o una evaluación de procesos y productos— que puede ser replicable o sólo aplicable a un caso singular;

e. Cuenta con un objetivo que debe estar justificado en un interés nacional, regional o local, sea por acciones estatales o privadas;

f. Debe presentar la resolución de problemas y/o necesidades incorporando innovaciones cognitivas; esto es, no se limita a la aplicación de procedimientos, rutinas, metodologías, hallazgos, afirmaciones de conocimiento, etcétera, ya codificados y normalizados en la base de conocimientos accesible localmente y que es propia de las disciplinas del proyecto, aunque estos elementos formen parte del mismo;

g. Debe identificar una o más organizaciones públicas o privadas que estén en capacidad de adoptar el resultado desarrollado;

h. Puede identificar una o más organizaciones públicas o privadas que demanden de manera concreta el resultado desarrollado;

i. Debe tener una o más instituciones financiadoras que proveerán, garantizarán o contribuirán a su financiamiento; $y$

j. Debe contar con una evaluación previa realizada en la institución que presenta el proyecto al Banco Nacional por especialistas o idóneos, que contemplará: 1) factibilidad técnica y económico-financiera o equivalente; 2) adecuación de los recursos comprometidos (humanos, infraestructura y equipamiento, y financiamiento); y 3) informes de avances sobre la ejecución del proyecto cuando corresponda.

El Ministerio de Ciencia, Tecnología e Innovación productiva ha establecido un Banco de PDTS que se encuentra en su página web desde octubre 2012.

Desde 2012, se han financiado más de 300 proyectos PDTS con participación de múltiples disciplinas e instituciones del complejo de ciencia y tecnología. 
Re-significaciones de los recursos institucionales de gobernanza de la «tercera misión» de las universidades: el caso de los Proyectos de Desarrollo Tecnológico y Social (PDTS) de Argentina

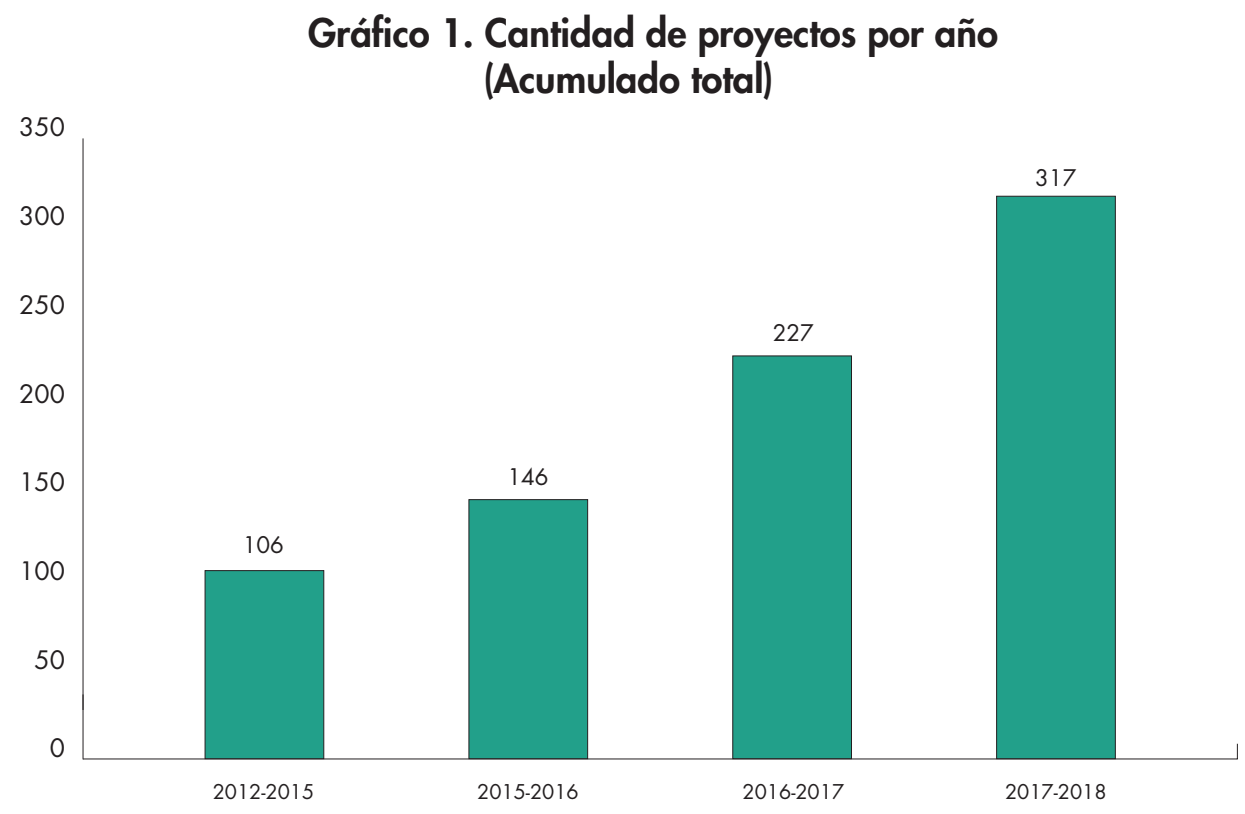

Fuente: elaboración propia en base a datos del MINCTIP.

\section{La pretensión de uso del conocimiento y su dimensión de análisis en la gobernanza de la tercera misión}

Zabala (2004) sostiene que existen tres niveles de análisis principales como fue planteado el problema sociológico de las relaciones entre producción y apropiación de conocimientos científicos: un nivel macrosocial donde la utilidad es pensada en términos históricos en relación el desarrollo del orden social, un nivel institucional o meso donde se analiza la forma en que los entornos institucionales alientan u obstaculizan los procesos de apropiación de los conocimientos, y un tercer nivel, de corte micro-social, que se centra en las interacciones siendo la utilidad el resultado de procesos concretos de apropiación, dialógicos y contingentes, entre agentes sociales. La utilidad social de los conocimientos científicos en tanto objeto analítico —en el plano meso sobre el que se hará énfasis - se encuentra mediada por la capacidad de determinadas instituciones dedicadas a la producción de conocimiento de convertir los mismos en recursos para el desarrollo. En efecto, el plano de análisis que se desprende de la articulación anterior supone entonces, siguiendo a Zabala (2004), concentrarse en «el comportamiento de determinadas instituciones en relación con un objetivo preciso: que los conocimientos producidos se conviertan en recursos para el desarrollo económico y social» (Zabala, 2004: 157). Esto supone observar de qué manera el contexto político e institucional incide en los procesos de construcción de utilidad de conocimientos científicos. Como se subrayó anteriormente, el enfoque teórico asume que las políticas públicas e institucionales, entendidas como dimensiones de contexto, pueden analizarse tanto como pautas y reglas externas a los actores a la vez que como elementos que forman parte de sus orientaciones de acción. Señalé que una mirada amplia del problema de la utilidad reconoce la existencia de pautas y reglas institucionalizadas en las comunidades científicas, en la toma de decisiones de políticas públicas o en los modos de funcionamiento de organizaciones que en parte ingresan en el escenario de interacción en el cual participan investigadores, intermediarios y usuarios. En ese espacio, a la vez que imponen límites a los modos de percepción y evaluación de los actores, también son pasibles de ser resignificados a la luz de la confrontación de cosmovisiones en el ambiente interactivo. Al respecto, los PDTS, sostengo se insertan en esta batería de esfuerzos institucionales por generar mecanismos para el desarrollo de conocimiento científico 
que sea «usado» (apropiado) por la sociedad, representada en alguna de las dimensiones institucionales que resignifica.

En líneas generales puede decirse que la orientación actual de las políticas de gobernanza de la «tercera misión» está fuertemente conectada con la idea reconocer trayectos diferenciados y como objetivo de máxima de fomentar y promover las innovaciones que eventualmente podrían derivarse del uso de los conocimientos generados por esos grupos. Este tipo de acciones impulsadas desde el MINCTIP explicitan una voluntad de generar instrumentos que articulen la producción académica con la resolución de problemas sociales. En el caso del instrumento PDTS, esas voluntades se convierten en recursos para el fin propuesto en dos sentidos: en primer lugar, habilitan a la institucionalización de una práctica de investigación que se reconoce como valiosa y promueven el desarrollo de capacidades en el sistema de gestión de CyT para identificarlas y, en segunda instancia, promoverlas.

\section{Recursos institucionales que los PDTS resignifican: Evaluación diferenciada y defini- ción de criterios de novedad local, relevancia, pertinencia y demanda del conoci- miento}

En efecto, tal como afirmé arriba, el objetivo de máxima de los PDTS quedó emparentado con la promoción de un mayor uso del conocimiento científico que se produce en el complejo de CyT nacional. Para la consecución de ese objetivo, el instrumento pone a disposición recursos (materiales y simbólicos).

Al plano material corresponden los recursos económicos asignados a las convocatorias (para financiamiento de proyectos y gestión de los mismos). En el plano simbólico, se ubican aquellas definiciones que, sostengo, son las más valiosas del instrumento y pueden servir como insumo en el futuro, dado el aprendizaje de la experiencia transcurrida.

En lo que sigue analizo seis dimensiones que el instrumento PDTS introduce y que analizaré en tanto recursos simbólicos (Bourdieu, 1979; Strauss, 1978) puesto que comienzan, luego de la implementación del instrumento PDTS a tomar parte del significado del instrumento de política científica que resignifican una práctica o conjunto de prácticas (la producción de conocimiento) que habilitan definiciones precisas de respecto del modo de un modo de producción de conocimiento orientado hacia fines de utilidad social. En efecto, estos recursos simbólicos (ver Tabla I) en el desarrollo del instrumento PDTS y su implementación resultan en capacidades en la medida en que redefinen o habilitan la institucionalización de prácticas individuales y colectivas. De este modo reconocer, definir e incorporar en el sistema de CyT un instrumento de política científica que propone definiciones sobre el modo de producción de conocimiento supone la capacidad de validar e institucionalizar prácticas que se consideran deseables y se buscan promover. 
Re-significaciones de los recursos institucionales de gobernanza de la «tercera misión» de las universidades: el caso de los Proyectos de Desarrollo Tecnológico y Social (PDTS) de Argentina

Tabla I. Dimensiones sobre las que los PDTS introducen resignificaciones

\begin{tabular}{c}
\hline Primera dimensión: evaluación diferenciada \\
\hline Segunda dimensión: criterio de novedad u originalidad local en el conocimiento \\
\hline Tercera dimensión: criterio de relevancia \\
\hline Cuarta dimensión: criterio de pertinencia \\
\hline Quinta dimensión: criterio de demanda \\
\hline
\end{tabular}

\subsection{Primera dimensión: evaluación diferenciada}

Sostuve que la introducción de una evaluación diferenciada para actividades de producción de conocimiento con fines de transferencia y uso es el elemento más distintito del instrumento PDTS. En efecto, recuperando la caracterización introducida en el por Fernandez Esquinas et al., (2011) respecto de los elementos constitutivos de la evaluación de actividades de Cy'T, analizo debajo aquellas redefiniciones en el instrumento PDTS sobre la evaluación y que, como dije más arriba, suponen recursos capaces de resignificar el modo de producción de conocimiento.

Tabla II. Redefiniciones de los elementos que componen la evaluación

\begin{tabular}{|c|c|c|}
\hline $\begin{array}{c}\text { ELEMENTO } \\
\text { DE LA EVALUACIÓN }\end{array}$ & $\begin{array}{c}\text { MODELO } \\
\text { TRADICIONAL* }\end{array}$ & $\begin{array}{l}\text { RECURSOS EN EL MARCO } \\
\text { DE LOS PDTS }\end{array}$ \\
\hline Elemento 1 & Autogobierno de las comunidades cientificas & Inclusión de actores extra-académicos en la evaluación \\
\hline Elemento 2 & $\begin{array}{l}\text { Separación funcional de las agencias de evaluación respecto a los organismos } \\
\text { de la política cientifica }\end{array}$ & Evaluación de resultados en instituciones \\
\hline Elemento 3 & Evaluación por pares ex ante & Evaluación cuatripartita \\
\hline Elemento 4 & Herramientas de financiación y evaluación dirigidas a individuos & $\begin{array}{l}\text { Evaluación dirigida a grupos que impactan en los informes y promociones } \\
\text { individuales de los investigadores involucrados }\end{array}$ \\
\hline Elemento 5 & $\begin{array}{l}\text { Evaluación de proyectos tipo «bottom-up» es decir a partir de proyectos } \\
\text { propuestos por los involucrados. }\end{array}$ & $\begin{array}{l}\text { Inclusión de nuevos insumos para la evaluación como son los informes de los } \\
\text { demandantes } 0 \text { adoptantes }\end{array}$ \\
\hline Elemento 6 & $\begin{array}{l}\text { Separación entre la evaluación científica y la evaluación del desarrollo } \\
\text { tecnológico. }\end{array}$ & $\begin{array}{l}\text { Evaluación basada en procesos y resultados por un coniunto de expertos que } \\
\text { acuerdan criterios diferenciados }\end{array}$ \\
\hline Elemento 7 & Outputs de la práctica de investigación: papers, libros, patentes, etc. & Outputs: resultados, grado de adopción, evaluación de procesos. \\
\hline
\end{tabular}

Fuente: elaboración propia.

* (Fernandez Esquinas et al., 2011).

- Elemento 1: Autogobierno de las comunidades científicas.

Siguiendo a Fernandez Esquina et al., (2011) son los propios científicos los que deciden cuáles son las pautas de evaluación (Fernandez Esquinas et al., 2011). Si bien existen tensiones en todo complejo de CyT respecto de la evaluación y ésta no está nunca desvinculada por completo del contexto en el que se inserta ${ }^{6}$, la «comunidad científica» cuenta con un alto grado de autonomía para definir los criterios de asignación de recursos.

En efecto, un punto a destacar en el proceso de evaluación de PDTS es la incorporación de nuevos actores en esta instancia. La participación de representantes de las entidades adoptantes y demandantes significó una apertura hacia la evaluación extendida (Jiménez-Buedo y Vielba, 2009: 732) y, de esta manera, la pertinencia y la relevancia de las investigaciones dejan de ser definidas por pares exclusivamente, de modo que el ejercicio supone una operación de apertura

6 El sistema de Cy'T requiere de inputs materiales, ligados a recursos económicos que el Estado le provee. Sin embargo, el proceso de asignación de esos recursos es mayormente definido mediante criterios establecidos por la propia comunidad científica. 
que discute el autogobierno imperante en el proceso de evaluación de la práctica científica ${ }^{7}$. La modalidad de evaluación conjunta genera mecanismos de interacción fructuosos desde distintas miradas, donde un técnico, idóneo o usuario (demandante u adoptante) interactúa con un evaluador par (disciplinar) para determinar la factibilidad técnica del proyecto.

- Elemento 2: Separación funcional de las agencias de evaluación respecto a los organismos de la política científica

El instrumento PDTS introdujo algunos matices novedosos en materia de evaluación de actividades de CyT respecto de la separación funcional de las agencias de evaluación. Si bien la evaluación de desempeño de los investigadores involucrados en proyectos PDTS continúa estando en la órbita de la pertenencia institucional de cada investigador, se generaron instancias intermedias de evaluación para las actividades PDTS para las que se crearon instancias institucionales específicas. El caso de las Comisiones de Acreditación al BNPDTS que pondera la capacidad de cumplimiento de objetivos y consecución de resultados. Por su parte la Comisión de Desarrollo tecnológico y social que evalúa el desempeño de un investigador en cuanto a su informe reglamentario y promoción en caso de investigadores de Conicet en el caso de dirigir un PDTS. En este sentido, se observan cambios en la modalidad de evaluación en dos niveles: en un primer nivel, en el que se evalúa el proyecto PDTS y en un segundo nivel en el que se evalúa el desempeño del investigador.

- Elemento 3: Predominio del sistema de evaluación por pares ex-ante.

En línea con lo descripto para el Elemento 1, en los PDTS se introduce, además de la función ex-ante, una función de evaluación ex-post en la que debieran participar actores externos al complejo de CyT. El aspecto relevante lo constituye la búsqueda de evaluadores, que, mientras que regularmente queda a cargo de las comisiones disciplinares que se encargan de la designación de pares externos, en esta instancia se amplía también al personal técnico de la del MINCTIP, que se encarga de analizar los temas de los proyectos y hacer búsquedas selectivas, por ejemplo, en colegios profesionales o cámaras empresarias. Es de destacar que muchas veces los evaluadores idóneos son los mismos pares (que para estos casos coincide con la definición de evaluador disciplinar).

La necesidad de realizar una evaluación presencial cuatripartita, a la vez que promueve la vinculación extra pares, complejiza la evaluación en términos organizativos, teniéndose que incorporar nuevas rutinas por parte de los distintos actores que participan en los procesos de evaluación, lo que exige un esfuerzo mayor de coordinación. A lo que efectivamente se ha recurrido es a la valoración del informe del demandante o adoptante del proyecto como insumo.

- Elemento 4: Herramientas de financiación y evaluación dirigidas a individuos.

En este nivel, los PDTS introducen un formato de evaluación que reconoce el trabajo en grupo inherente a la práctica académica. Mientras que las evaluaciones de proyectos y su financiación están principalmente abocadas a identificar la capacidad del director de proyecto y del equipo de producir «productos» publicados, el foco en el caso de los PDTS está puesto en el proyecto, su proceso y resultados y en el grupo de trabajo ponderando los roles e impactando en la evalua-

\footnotetext{
Este tipo de evaluaciones está siendo implementada de manera experimental en algunas experiencias como las llevadas a cabo recientemente en Barcelona donde el Ministerio de Salud incluyó a beneficiarios en la evaluación de proyectos de investigación desde allí promovidos.
} 
ción individual. Suponen en este sentido, un recurso en la dirección de complejizar la evaluación de la práctica científica reconociéndola como producto de una labor colectiva. La pretensión manifiesta de los que promueven el instrumento desde la secretaría de CyT es que también la participación en PDTS impacte en los concursos docentes a través del reconocimiento de este tipo de prácticas investigativas ${ }^{8}$.

- Elemento 5: Separación entre la evaluación científica y la evaluación del desarrollo tecnológico. El instrumento de evaluación para PDTS supone que la evaluación científica y la tecnológica no son a priori dos estadios diferenciados que deban ser evaluados por separado. Incluso cuando esto no necesariamente estuvo presente en el origen del instrumento, más bien orientado a dar marco a actividades tecnológicas, el resultado supone un mecanismo en el que esta diferenciación no presenta un peso significativo o bien no resulta definido previo a la evaluación del proyecto.

Siguiendo a Roca y Versino (2010), en la evaluación disciplinar, por pares: «la pertinencia se subsume a la calidad y la excelencia a la evaluación de pares garantizando el funcionamiento de los mecanismos de reproducción de conocimientos que históricamente han impedido concebir actividades de investigación y desarrollo en función de objetivos adecuados a las necesidades locales». (Versino y Roca, 2010: 10). En la evaluación PDTS se atiende a criterios de acreditación que califican a los proyectos en función de su aporte a la resolución de problemas, necesidades o demandas identificables en la sociedad y/o expresadas por los agentes sociales en la esfera de la política, el mercado, el territorio, la cultura o la estructura social, en sentido amplio. La característica de dicho aporte en materia de conocimiento es lo que permitirá identificar a un PDTS y diferenciarlo de otras actividades científico-tecnológicas y profesionales que no se incluirían en la misma categoría de actividad.

- Elemento 6: Predominio de procedimientos de evaluación de tipo «bottom-up» para la asignación de recursos a proyectos.

Si bien el modelo bottom-up no presenta en el instrumento PDTS cambios significativos, puesto que son los propios investigadores los que presentan los proyectos, sí puede observarse un matiz que propone una vía alternativa en el proceso de incorporación de demandas al involucrar un adoptante y demandante en la formulación del proyecto. En un segundo nivel, los PDTS incorporan el mecanismo de financiamiento externo con la figura de institución financiadora, por lo que también permiten incorporar a la «demanda», traducida en financiamiento, para reconocer una evaluación diferenciada que no solamente sea originada en el ámbito académico.

- Elemento 7: Outputs de la práctica de investigación.

Un último elemento respecto de la evaluación diferenciada que proponen los PDTS, que no se corresponde directamente con el esquema presentado por Fernandez Esquinas et al., (2011) supone el reconocimiento de otros productos (outputs) posibles de la práctica científico-tecnológica. Mientras que, mediante la evaluación tradicional, como sostuvimos antes, pone su énfasis en productos mensurables de la investigación (publicaciones, patentes, contratos, etc.) la evalua-

8 En la actividad llevada a cabo en el mes de septiembre de 2019 en la Universidad de Buenos Aires se expresó la voluntad de promover que en los concursos docentes los PDTS permitan valorar este tipo de investigaciones y también se han reconocido los PDTS como proyectos que perciben el Incentivo Docente otorgado por la Secretaría de Políticas Universitarias a docentes universitarios investigadores. 
ción PDTS supone un recurso para reconocer la existencia de otros productos posibles, ligados a resultados o al cumplimiento de objetivos que no se inscriben en los indicadores tradicionales y para los cuales son necesarias una batería de capacidades de evaluación que no se encuentran todavía maduras ${ }^{9}$. En este nivel resulta sustantiva el insumo que ofrezca el demandante y adoptante (materializado en una grilla de cumplimiento de objetivos) sobre la que se cimenta la evaluación de desempeño del proyecto.

\subsection{Segunda dimensión: criterio de novedad u originalidad local en el conocimiento} Un proyecto PDTS, necesariamente, debe pretender generar un aporte de nuevos conocimientos. Ahora bien, mientras que la referencia a la originalidad supone el desarrollo de conocimiento «nuevo» en tanto descubrimientos que «corran las fronteras del conocimiento» en el marco de los PDTS, mediante una redefinición del concepto, se incorpora lo local, en tanto contexto de aplicación y demanda, como garante de originalidad.

Las consideraciones sobre la originalidad de los proyectos sugieren la necesidad de revisar el concepto de novedad del conocimiento a un significado acotado a las condiciones locales: se trata, entonces, de entender la cuestión de la originalidad en estrecha relación con lo local de ese conocimiento.

Esta operación de resignificación, de corte epistemológica, propone un matiz de resolución respecto de las discusiones sobre ciencia básica, ciencia aplicada y desarrollo tecnológico. Si se hace foco en el proceso de producción de conocimiento, situado y contextual, la originalidad o novedad puede emparentarse a la capacidad de reformular un problema de conocimiento o realizar traducciones para su aplicación lo que, en un contexto determinado, en el marco de un PDTS, puede ser definido como original.

\subsection{Tercera dimensión: criterio de relevancia}

El criterio de relevancia, más que cualquier otro, está relacionado con los objetivos de la política pública, el sentido de urgencia o necesidad de la problemática a resolver mediante el conocimiento aplicado o el impacto esperado de los resultados de la innovación tecnológica. En efecto, la relevancia es un concepto estrictamente político (en sentido amplio) y, por lo tanto, no califica a características intrínsecas de los proyectos de investigación sino a los objetivos o fines a los que tiende y a los objetos a los cuales se aplican. Una referencia directa a la relevancia es la adecuación de los objetivos del proyecto a las políticas públicas o a los objetivos estratégicos tanto de la política de ciencia y tecnología (nacional, provincial o municipal) como de políticas de otros sectores que inciden en la producción de conocimientos (políticas de salud, industrial, agropecuaria, de vivienda, etcétera). Tal como hemos reconocido luego de haber indagado y propuesto definiciones diversas sobre los criterios de pertinencia y relevancia los documentos que dan origen a los PDTS son los primeros en los que se diferencian de forma explícita desde un documento oficial (Naidorf, Vasen, Alonso, 2016).

Pero también la relevancia puede referir a puntos de vista diferentes a los de la política pública y vincularse a objetivos de política de sectores de la sociedad civil o a valores más o menos generalizados en la sociedad. Esto puede generar contradicciones entre diferentes orientaciones que se desenvuelven en ella y que se pueden manifestar en los objetivos de los proyectos.

9 Son los propios evaluadores entrevistados los que demandan mayores especificaciones e instrumentos para la toma de decisiones. 
La resolución del criterio de relevancia está necesariamente en manos de las instituciones que presentan los PDTS al Banco de proyectos de acuerdo a las prioridades que expresan las mismas en cuanto a su relación con la sociedad. En este sentido, la relevancia para los PDTS, reconocida como un concepto estrictamente político, representa un corrimiento del ideal de relevancia de la investigación, a crítica y multívoca, sobre la que se construyen discursos para legitimar prácticas de investigación. La relevancia, está, en el marco de los PDTS, contextualmente situada y explícitamente definida para cada PDTS en tanto resulta de una atribución de los agentes que intervienen en la producción de conocimiento.

\subsection{Cuarta dimensión: criterio de pertinencia}

Si la relevancia indica la importancia de los objetivos del PDTS en relación a las necesidades y urgencias de la sociedad, expresas por los agentes que la definen, la pertinencia considera si la estrategia de investigación y la metodología propuestas por el proyecto, como así también los resultados esperados son adecuados para resolver el problema identificado en la localización o para el uso concreto postulado por el proyecto. Este es un criterio que refiere, entonces, a condiciones «internas» del proyecto con las condiciones «externas» de la aplicación de sus resultados. El análisis de la pertinencia es próximo al de la capacidad del proyecto de alcanzar los resultados esperados, un análisis que considera a la metodología, recursos disponibles, accesibilidad al objeto de estudio, adecuación de recursos humanos, etcétera que es típico de todo proyecto (básico o aplicado). Si este análisis se refiere solamente a componentes internos del proyecto, el de la pertinencia contrasta estos componentes con el objetivo y el contexto de la aplicación de sus resultados. Al igual que en el caso del criterio de relevancia, la consideración del criterio de pertinencia en cada proyecto está a cargo de los agentes que lo definen.

\subsection{Quinta dimensión: criterio de demanda}

Una condición clave de los PDTS es la existencia de un agente demandante, un agente adoptante y un agente financiador del proyecto. En todas las áreas de conocimiento, la existencia de demanda constituye evidencia de que los resultados del proyecto interesan a la sociedad, representada en un actor institucionalizado, y no solamente a la comunidad de investigación desde un punto de vista del interés disciplinar.

El concepto de demanda sugiere la acción de requerir un aporte o bien determinado a los organismos de ciencia y tecnología y universidades sea a sus autoridades, estructuras burocráticas o a través de sus investigadores. Si bien la noción de demanda no es para nada nueva en el marco de las PCTI, significan un intento sostenido por incorporar actores en el proceso de organización de agendas de investigación. En este sentido, incluso cuando otros instrumentos de política de CyT ya contaran con alguna definición respecto de la demanda de conocimiento, estos estaban mayormente orientados al plano productivo y vinculados a empresas. En el caso PDTS, se reconoce, también, como «un otro» en proceso de producción de conocimiento no solamente empresas sino también otro tipo de organizaciones de la sociedad civil e incluso, como se dijo antes, dependencias del Estado (en todos sus niveles).

En este sentido, representan también un impulso por definir y dar significado preciso a la inclusión (y modalidad) de actores externos al proceso de producción de conocimiento. 
Estas dimensiones analizadas suponen un proceso de resignificación del modo de producción de conocimiento valioso y novedoso en algunos casos en vías de dar marcos institucionales a nuevas prácticas y perfiles de investigación e investigadores.

Puesto que, como se dijo antes, la cultura académica (Naidorf, 2005) supone un conjunto de prácticas y definiciones sobre el quehacer académico que habilita y constriñe cursos posibles de acción, los esfuerzos por dar marco institucional (y legitimidad) a actividades que no se reconozcan como hegemónicas dentro de la propia cultura académica son valiosos en tanto plausibles de promover su reproducción.

\section{Discusión}

Más allá de los alcances y limitaciones en su implementación, analizados en otros trabajos (Naidorf et al., 2018; Senejko, 2019; Naidorf et al.; 2020, entre otros), los PDTS cuentan con el objetivo de máxima, enunciado en el propio Documento I, de: «hacer uso del conocimiento científico-tecnológico (...) y tienen por objetivo la resolución de problemas o necesidades de carácter práctico» (Documento I: 1).

Las definiciones de uso que proponen los PDTS, en tanto instrumento de política científica, apuntan a promover espacios de interacción entre científicos y usuarios, ofreciendo mecanismos de evaluación diferenciada a los investigadores para tal fin y esto es, para todos los casos de política científica, un ejercicio de delegación, en cuyo seno se ubican las asimetrías de información entre quienes señalan un marco de acción y los agentes que se los apropian, rechazan o resignifican, lo que, en efecto, se observa en el bajo grado de participación de los investigadores en el instrumento y la incluso menor participación en su dimensión más innovadora: la evaluación diferenciada.

Los agentes políticos a cargo de la gobernanza de estas actividades, ponen a disposición recursos materiales, como el financiamiento específico de proyectos y simbólicos, asociados a la gestión de una evaluación diferenciada que, se suponía, sería capaz de impulsar el desarrollo de proyectos que se incorporen al BNPDTS.

Los PDTS introducen una nueva modalidad que buscó, en algún sentido, empujar al investigador a incorporar al destinatario de su producción en el proceso de producción de conocimiento, asumiendo que producto de esta relación resultaría un mayor uso del conocimiento producido ${ }^{10}$. En la propuesta del primer diseño de los PDTS, ésta se orientó principalmente a académicos con perfiles «mixtos», que procuran «mantenerse» en el sistema y no ser penalizados si hacen investigación orientada que no resulte en los outputs tradicionales que el sistema evalúa favorablemente.

Por su parte, la figura del actor externo fue ampliándose progresivamente por iniciativa de las universidades. Tal es así que fueron las universidades las que propusieron que en lugar de denominarse Proyectos de Desarrollo Tecnológico también se agregaría la categoría «social» para no asociarlo solo a vinculación Universidad-Empresa.

La importancia de incluir a actores externos en el diseño, implementación y evaluación de proyectos es nuestra preocupación desde larga data (Naidorf, 2009; Vasen, 2013) y se había comenzado a reflejar desde

10 Vale aclarar que junto con los PDTS el programa PROCODAS (Programa Consejo de la Demanda de Actores Sociales creado por Resolución Ministerial N. ${ }^{\circ}$ $609 / 2008$ también se propuso incorporar al destinatario final como parte del proceso de producción de conocimiento. Acerca de los alcances del programa se puede consultar: Galante 2009 y Estébanez 2011. 
Re-significaciones de los recursos institucionales de gobernanza de la «tercera misión» de las universidades: el caso de los Proyectos de Desarrollo Tecnológico y Social (PDTS) de Argentina

lo normativo en la Ley de Educación Superior a partir del denominado «consejo social», para el caso de las universidades, pero aún hoy presenta dificultades su efectiva implementación.

Más allá de estas dificultades, el análisis contextuado del surgimiento del instrumento y de sus implicancias en materia de investigación académica, muestran un esfuerzo por institucionalizar mecanismos de vinculación efectiva entre universidad y sociedad, reconociendo el significado virtuoso de esta vinculación en tanto actividad de tercera misión de las universidades.

Quizás el análisis de la experiencia PDTS pueda servir como insumo también para repensar gobernanza de la ciencia y la tecnología, la dinámica de sus actores, sus fortalezas, debilidades y aquellos ámbitos en los que desarrollar dependencias institucionales que sean las responsables de promover procesos de uso de conocimiento para consolidar aquella función social vinculada la tercera misión que de la universidad se espera.

\section{Referencias bibliográficas}

Albornoz, Mario y Ariel Gordon (2011): "La política de ciencia y tecnología en Argentina desde la recuperación de la democracia (1983-2009)" en Mario Albornoz y Jesús Sebastián (eds.): Trayectorias de las politicas cientificas y universitarias de Argentina y España. Madrid: CSIC.

Benneworth, Paul Stephen (2014): “Decoding University Ideals by Reading Campuses”. The Physical University, 217-242.

Bourdieu, Pierre (1979): “Symbolic Power". Critique of Anthropology, 4(13-14), 77-85.

Bourdieu, Pierre (1984): “Homo Academicus”. Buenos Aires: S.XXI, 2008.

Di Bello, Mariana \& Romero, Lucía Ana (2018): "Vinculación y extensión universitaria: la relación entre la universidad y sus entornos en las universidades nacionales de Quilmes y Lanús”. Apuntes, 45 (82), 145-171.

E3M Project. (2012). Green Paper: Fostering and Measuring «Third Mission» in Higher Education Institutions.

Etzkowitz, Henry (1990): “The Second Academic Revolution: The Role of the Research University in Economic Development” en Susan E. Cozzens, Peter Healey, Arie Rip y John Ziman (eds): The Research System in Transition. Springer: Dordrecht. DOI: https://doi.org/10.1007/978-94-009-2091-0_9.

Etzkowitz, Henry y Leydesdorff, Loet (2000): “The Dynamics of Innovation: from National Systems and 'Mode 2' to a Triple Helix of University-Industry-Government Relations'. Research Policy, 29 (2), 109-123.

Fernández-Esquinas, Manuel; Torres-Albero, Cristóbal; Rey-Rocha, Jesús y Martín-Sempere, María José (2011): "Dissemination Practices in the Spanish Research System: Scientists Trapped in a Golden Cage". Public Understanding of Science, 20 (1), 12-25.

Fischman, Gustavo (2014): "If the Research is Not Used, Does it Exist". Teachers College Record, 17570, 1-10.

Geuna, Aldo y Muscio, Alessandro (2009): “The Governance of University Knowledge Transfer: A Critical Review of the Literature". Minerva, 47 (1), 93-114. 
Hayden, Markus C.; Weiß, Martin; Pechriggl, Alice y Wutti, Daniel (2018): Insights into University Knowledge Transfer in the Social Sciences and Humanities (SSH) and Other Scientific Disciplines-More Similarities Than Differences. Frontiers in Research Metrics and Analytics, 3, 32. DOI: https:// doi.org/10.3389/frma.2018.00032.

Jasanoff, Sheila (2003). States of Knowledge: The Co-Production of Science and Social Order. Londres: Routledge.

Kreimer, Pablo y Thomas, Hernán (2004). Producción y uso social de conocimientos. Estudios de sociología de la ciencia y la tecnología en America Latina. Bernal: Universidad Nacional de Quilmes.

Laredo, Philippe (2007): "Revisiting the Third Mission of Universities: Toward a Renewed Categorization of University Activities?”. Higher Education Policy, 20 (4), 441-456.

Lockett, Andy; Wright, Mike y Wild, Andrew (2013): "The Co-Evolution of Third-Stream Activities in UK Higher Education”. Business History, 55 (2), 236-258.

Lundvall, Bengt-Åke (1992): “User-Producer Relationships, National Systems of Innovation and Internationalisation" en Bengt-Åke Lundvall (eds.): National Systems of Innovation: Towards a Theory of Innovation and Interactive Learning. Londres: Pinter Publishers.

Merton, Robert K. (1937): “The Sociology of Knowledge”. Isis, 27 (3), 493-503.

Meyer-Thurow, Georg (1982): "The Industrialization of Invention: A Case Study From the German Chemical Industry”. Isis, 73 (3), 363-381.

Molas-Gallart, J., \& Castro-Martínez, E. (2007). Ambiguity and conflict in the development of 'Third Mission 'indicators. Research Evaluation, 16(4), 321-330.

Molas-Gallart, Jordi; Salter, Ammon; Patel, Pari; Scott, Alister y Duran, Xavier (2002). Measuring Third Stream Activities. Final Report to the Russell Group of Universities. Brighton: SPRU, University of Sussex.

Naidorf, Judith (2010) Mercantilización de la Universidad Primer Foro Nacional de Educación para el Cambio Social (1 $1^{a}$ ed.). Buenos Aires: El Colectivo, 2010. Encuentro Nacional de Estudiantes de Organizaciones de Base (ENEOB).

Naidorf, Judith C. (2014): “Knowledge Utility: From Social Relevance to Knowledge Mobilization”. Education Policy Analysis Arcbives, 22, 89.

Naidorf, Judith; Vasen, Federico y Alonso, Mauro (2016): "Los proyectos de desarrollo tecnológico y social como política científica”. Brazilian Journal of Latin American Studies (PROLAM/USP), 27.

Naidorf, Judith; Perrotta, Daniela; Gómez, Sebastián y Riccono, Guido (2015): "Políticas universitarias y políticas científicas pos 2000. Crisis, innovación y relevancia social”. Revista Cubana de Educación Superior. Número Especial, 10-28.

Naidorf, Judith; Vasen, Federico y Alonso, Mauro (2019). "Aunar criterios en un sistema fragmentado. tensiones en torno a evaluación de la investigación aplicada y el desarrollo tecnológico en el origen de los proyectos de desarrollo tecnológico y social”. Eccos, (49), 1-21. 
Naidorf, Judith (2003): “La privatización en Universidades públicas” en P. Gentili y B. Levy (eds.): Espacio público y privatización del conocimiento. Buenos Aires: Clacso, 2005.

Nelson, R. R. y Rosenberg, N. (1993): “Technical Innovation and National Systems. National Innovation Systems: A Comparative Analysis”. Oxford University Press, 7, 1-18.

Nowotny, Helga; Limoges, Camille y Gibbons, Michael (1994). The New Production of Knowledge: The Dynamics of Science and Research in Contemporary Societies. Londres: Sage Publications.

Olmos-Peñuela, Julia (2015): “Scientists' Engagement in Knowledge Transfer and Exchange: Individual Factors, Variety of Mechanisms and Users”. Science and Public Policy, 45 (6), 790-803.

RICYT (2013). Red de Indicadores de Ciencia y Tecnología. Indicadores deficiencia y tecnología en Iberoamérica. www.ricyt.org.

Sánchez-Barrioluengo, Mabel (2014): “Articulating the 'Three-Missions' in Spanish Iniversities”. Research Policy, 43 (10), 1760-1773.

Schoen, Antoine y Theves, Jean (2006). Strategic Management of University Research Activities, Methodological Guide. Lugano: PRIME Project 'Observatory of the European University.

Senejko, María Paula y Versino, Mariana (2018): “La producción de conocimientos y la resolución de problemas sociales: Análisis de las convocatorias a proyectos de investigación orientados en la UBA (2003-2015)". Revista Horizontes Sociológicos, Asociación Argentina de Sociología.

Strauss, Anselm L. (1978). Negotiations: Varieties, Contexts, Processes, and Social Order. San Francisco: Jossey-Bass Inc Pub.

Trencher, Gregory; Yarime, Masaru; McCormick, Kes; Doll, Christopher N. H.; Kraines, Steven y Kharrazi, Ali (2014): "Beyond the Third Mission: Exploring the Emerging University Function of Co-Creation for Sustainability". Science and Public Policy, 41 (2), 151-179.

Vaccarezza, Leonardo Silvio y Zabala, Juan Pablo (2002). La construcción de la utilidad social de la ciencia: Investigadores en biotecnología frente al mercado. Bernal: Universidad Nacional de Quilmes Ediciones.

Weiss, Carol Hirschon (1999): “The Interface between Evaluation and Public Policy". Evaluation, 5 (4), 468-486. DOI: https://doi.org/10.1177/135638909900500408.

Wutti, Daniel y Hayden, Markus C. (2017): “Knowledge Transfer in the Social Sciences and Humanities (SSH) - Definition, Motivators, Obstacles, and Visions”. Colloquium: New Philologies, 2 (1). DOI: https://doi.org/10.23963/cnp.2017.2.1.7.

Zaharia, Sorin E. y Gibert, Ernest (2005): “The Entrepreneurial University in the Knowledge Society”. Higher Education in Europe, 30 (1), 31-40.

\section{Nota biográfica}

Mauro Alonso es licenciado en Sociología. Magíster en Estudios Sociales de la Ciencia y la Tecnología, por la Universidad Nacional de Quilmes (UNQ). Actualmente es becario doctoral del Consejo Nacional 
de Investigaciones Científicas (CONICET), en el Programa de Investigación de Sociología de la Educación del Instituto de Ciencias de la Educación, de la Facultad de Filosofía y Letras de la Universidad de Buenos Aires (UBA). Desarrolla su trabajo en las áreas de Psicología y Ciencias de la Educación y de Sociología de la Educación, siendo su especialidad los estudios sobre la universidad y los estudios sociales de la ciencia. El tema específico de su tesis doctoral, dirigida por la Doctora Clara Judith Naidorf, es: La utilidad social de la investigación en ciencias sociales: análisis de los Proyectos de Desarrollo Tecnológico y Social (PDTS). 